\title{
Considerations for the adoption of a Mediterranean diet beyond the Mediterranean Sea: lessons from Australian trials
}

\section{Abstract}

The Blue Zones are known for healthy longevity and low rates of chronic disease. Common denominators between blue zones include social, environmental and spiritual foundations for good health, however there are key dietary contributors including mindful eating and a predominantly plant-based diet. the Mediterranean diet (MedDiet) is a plant-based diet and is reported to reduce the risk of overall mortality and cancer incidence, diabetes, neurodegenerative disease, heart attack and cardiovascular disease (CVD). Populations have enjoyed the health benefits of a MedDiet, for years, which could offer better health to Western countries which suffer from lifestyle diseases like obesity and heart disease. But how feasible is it to implement such a pattern beyond the Mediterranean sea? The MedLey study explored whether a MedDiet could be adhered to for 6 months and would improve CVD risk factors compared with habitual diet (HabDiet) in a population of older Australian adults. Volunteers were assessed at 3 points during the study (0, 3, 6 months) and 12 months after the intervention had finished (18 months). 137 volunteers completed the trial and 128 volunteers completed the follow-up study. Participants completed a food frequency questionnaire and a 15-point MedDiet adherence score (MDAS; greater score $=$ greater adherence) was calculated. Home BP was measured over 6 days, and cardiometabolic health outcomes were assessed. Data were analysed using intention-to-treat LMEM with a group x time interaction term comparing data at 0, 3, 6 and 18-months (12-months post-trial). At baseline the MedDiet score was $6.7 \pm 0.2,9.6 \pm 0.2$ at 4 months and $7.9 \pm 0.3$ at 18 -months $(\mathrm{p}<.0001$ to baseline and 4 months). The MedDiet resulted in improved systolic BP, endothelial dilatation, oxidative stress and plasma triglycerides in comparison with HabDiet, after 6 months $(\mathrm{p}<.05)$. These changes were not sustained at $18-\mathrm{months}$ but did not completely return to baseline values. Principles of the MedDiet appeared to be somewhat maintained. Consumption of olive oil, legumes, fish and vegetables $(\mathrm{p}<.01)$ remained higher and discretionary food consumption $(\mathrm{p}=.02)$ remained lower at 18 -months than baseline in the MedDiet group. We have shown that following a MedDiet for 6-months is feasible and results improvements in markers of CVD risk. Some principles of the MedDiet were maintained following trial completion, but ongoing support may be helpful in maintaining MedDiet adherence and improved health. To implement such a pattern on a population level, several considerations are required including provision of resources and information, ongoing support, creating supportive environments through a multi-settings approach.

\section{Conflict of Interest}

There is no conflict of interest 